\title{
Donor-Site Lymphedema Following Lymph Node Transfer for Breast Cancer-Related Lymphedema: A Systematic Review of the Literature
}

\author{
Efterpi Demiri, MD, PhD, Dimitrios Dionyssiou, MD, PhD, Antonios Tsimponis, MD, PhD, \\ Olga Christina Goula, MD, PhD, Panagiotis Mılothridis, MD, Leonidas Pavlidis, MD, PhD, \\ Georgia Alexandra Spyropoulou, MD, PhD, and Perikles Foroglou, MD, PhD
}

\begin{abstract}
s
Background: Among current surgical options used for treating breast cancer-related lymphedema (BCRL), autologous lymph node transfer (ALNT) is shown to provide favorable results. However, postoperative donorsite lymphedema (DSL), following the lymphatic flap harvesting from the groin area, has already been reported. Our aim is to summarize the recent literature for evidence of DSL following an ALNT for BCRL.

Methods and Results: A PubMed bibliographic search was performed for published studies evaluating donor-site complications following LNT in BCRL patients. We recorded demographic data of the patients, the type of flap used, the follow-up, the donor-site morbidity, and the diagnostic tests performed pre- and postoperatively. Statistical analysis was conducted to document any correlation between the incidence of DSL and the abovementioned recorded parameters. According to our results, 11 studies met the inclusion criteria. From a total of 189 patients, three cases with DSL of the lower limb were reported (1.6\%). No statistically significant correlations were found.

Conclusion: ALNT has become increasingly popular and is considered an effective surgical option for treating BCRL of the upper limb. Although the incidence of postoperative DSL is low, insufficient data on patients' demographics, surgical details, and postoperative assessment do not allow extracting significant correlations. Meticulous technique of lymph node harvesting should be seriously considered to further minimize this infrequent but debilitating complication.
\end{abstract}

Keywords: donor-site, lymphedema, lymph node

\section{Introduction}

B REAST CANCER-RELATED upper limb lymphedema consists a significant consequence following breast cancer treatment and affects more than $30 \%$ of the patients. ${ }^{1} \mathrm{Lymph}$ node dissection, radiotherapy, abundant scar formation in the axilla, all constitute major risk factors for developing this most debilitating complication. ${ }^{2}$ Obesity, infection, and trauma are also considered significant risk circumstances for the development of secondary lymphedema, whereas genetic, as well as other unknown mediators may play an important role. ${ }^{3-7}$

Conservative management has been the mainstay of lymphedema treatment for several years, having uniquely evolved during the last decades. ${ }^{8}$ However, recurrent lymphedema and infection episodes and the need for patients' lifetime com- mitment incubated new revolutionary and promising methods of treatment, namely microsurgical procedures. ${ }^{9}$ Lymphaticovenous anastomosis, lymphatic-venous-lymphatic shunts, and lymph node transfer (LNT) gained popularity during the last three decades and are considered effective surgical procedures for treating lymphedema, especially when conventional therapeutic options fail to offer a permanent solution. ${ }^{10,11}$

It is widely recognized that autologous transplantation of vascularized lymph nodes may improve lymphatic drainage of an affected limb in patients who were found to have damaged lymph nodes or hypoplastic lymphatic vessels. ${ }^{12,13}$ LNT was first described by Chen et al., as a method to treat obstructive lymphedema in animal models. ${ }^{14}$ In 1982 Clodius et al., reported the first clinical application of autologous

Department of Plastic Surgery, Faculty of Health Sciences, School of Medicine, "Papageorgiou" Hospital, Aristotle University of Thessaloniki, Thessaloniki, Greece. 
vascularized lymph node transfer ${ }^{15}$; thereafter, encouraging results from various centers worldwide have established the above technique. However, its expanding use over the last years raised great concern about the possible complications that may appear over the area of lymph node harvesting. Seroma, lymphocele, lymphorrhea, infection, delayed wound healing, or wound dehiscence are the most common early postoperative complications, which are usually managed successfully without further implications. Chronic donor-site pain and lymphedema of the ipsilateral lower limb, have also been reported; these late complications are usually more persistent and sometimes irreversible. ${ }^{16}$

Although numerous studies have shown the effectiveness of lymph node transplantation in treating secondary lymphedema, very few published data systematically analyze the technique's overall complication rates, especially related to lymph node harvesting. ${ }^{17}$ The purpose of this study is to review the existing literature for the incidence of donor-site lymphedema (DSL) following autologous LNT (ALNT) in patients with breast cancer-related lymphedema (BCRL), focusing to elucidate the possible etiologic role of various parameters, including patients' demographics and lymph node harvesting techniques.

\section{Materials and Methods}

\section{Literature review}

Published data in peer-reviewed articles evaluating the incidence and/or complications at the donor site of a lymph node flap in lymphedema patients were analyzed. The bib- liographic search comprised the PubMed database (U.S. National Library of Medicine) for English literature published until June 2016. The following keywords were used: "lymph node transfer" OR "lymph node transplantation" AND "donor-site lymphedema" OR "lymphedema."

\section{Study selection}

Abstracts involving treatment of BCRL with ALNT were reviewed by two independent investigators; only those that provided data on complications of the above method were eligible for inclusion.

Experimental studies in animal models, anatomical studies, and studies reporting on primary lymphedema cases or secondary lymphedema of the lower limb were excluded from our research (Fig. 1).

\section{Data extraction}

Relevant studies were further searched for the following data, if available: patients' number, age and body mass index (BMI), type and main pedicle of the flap used, number of transferred lymph nodes, overall donor-site morbidity and complications related to lymph node harvesting, the followup period, and the imaging tests that were performed to evaluate the lymphatic status of the donor site before and after the operation.

\section{Statistical analysis}

Statistical analysis was conducted through SPSS (edition 17) software package. Kolmogorov-Smirnov was used to test

Relevant studies screened (Pubmed)

$\mathrm{N}=133$

Studies excluded (experimental studies, anatomical studies, studies reporting on

primary lymphoedema or secondary lymphoedema of the lower limb)

$\mathrm{N}=121$

Full text articles assessed for eligibility

$\mathrm{N}=12$

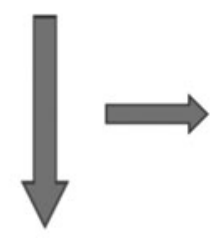

Studies excluded (no clear distinction of the sites related, insufficient data)

$N=1$

Studies finally included

$\mathrm{N}=11$

FIG. 1. Selection of the relevant included studies. 
normality for the quantitative variables (i.e., age, BMI, follow-up period). Data were thoroughly examined and independent samples $t$-test after Levene's test for equality of variables were used to figure out a correlation between the incidence of DSL and age. For the nonparametric variables (BMI and follow-up period), Mann-Whitney test was used. Lastly, to examine the correlation between the DSL and the flap type or the main pedicle, as well as the overall donor-site complication rate, Fisher's exact test was used. The level of statistical significance was determined to $p$-values $<0.05$.

\section{Results}

The initial search yielded 133 articles. Eventually and after a methodological evaluation, 12 studies were identified: one randomized control study, ${ }^{18}$ and 11 case series. ${ }^{8-10,12,16,19-24}$ Of those, one article, where Becker et al., ${ }^{12}$ describing the microlymphatic techniques for treating iatrogenic lymphedema publishing the largest series of ALNT in 1500 patients, was excluded, as there was no clear distinction of the sites treated; however, in another study by Vignes et al., ${ }^{16}$ which met the inclusion criteria, there is a clear statement that their material is a part of the total number of patients treated by C. Becker during the same period.

Another article, which is a case report of a donor-site lower limb lymphedema, ${ }^{24}$ provided some data on a series of BCRL patients, and thus this article was taken into consideration.

Therefore, a total of 11 studies were finally included and assessed in this systematic review (Table 1).

\section{Demographic data}

Overall, a total of 189 individuals (range 8-42 patients per study) were treated with a vascularized groin lymph node flap for breast cancer-related upper limb lymphedema. Patients' age ranged from 22 to 80 years with a body mass index ranging from 23.8 to $33.7 \mathrm{~kg} / \mathrm{m}^{2}$.

\section{Type and pedicle of the flap}

An inguinal lymph node flap was used in all cases; in most studies, lymph nodes together with a variable amount of the surrounding soft tissue from the groin area were harvested and transferred to the affected axillary region. In five studies, this lymph node flap was combined with a free abdominal flap, DIEP, or muscle-sparing TRAM flap, for simultaneous breast reconstruction (Table 1). Overall, in 59 patients, a combined procedure was performed, whereas in 122 cases a vascularized LNT alone was executed. In one study by Granzow et al., ${ }^{10}$ from a total of eight vascularized LNTs, the exact number of combined flaps is not reported.

In 7 out of 11 studies, the authors describe the technique for harvesting the lymph groin flap and the pedicle that was used; the superficial circumflex iliac artery and vein (SCIA$\mathrm{V})$ were recorded to be used in 87 patients, whereas the use of the superficial inferior epigastric artery and vein (SIEA-V) is reported in 29 cases.

In some series, where ALNT was associated to breast reconstruction with an abdominal flap, the vessels nourishing the lymph node flap were not systematically anastomosed to the recipient site, and the combined flap was based on the anastomosis of the abdominal flap pedicle. ${ }^{10,23}$

\section{Number of lymph nodes}

In most studies, information about the number of lymph nodes contained in the lymphatic tissue flap is not reported. In only two studies, the authors described in detail their harvesting technique and identified one to three lymph nodes per lymph flap ${ }^{18,21}$; in another study, where a DSL case is presented, the authors reported that the transferred flap included at least three inguinal nodes embedded in the surrounding fat tissue. $^{24}$

\section{Follow-up period}

The mean follow-up period reported was 37.97 months, ranging from 6 to 132 months postoperatively.

\section{Donor-site complications}

In eight studies, postoperative donor site complications are reported and included seroma $(n=8)$, lymphocele $(n=3)$, lymphorrhea $(n=2)$, wound infection $(n=2)$, delayed wound healing $(n=3)$, donor-site pain, numbness or discomfort $(n=9)$, transient edema of the donor site $(n=1)$, and lymphedema of the lower limb $(n=3)$. In 4 out of 11 studies, donor-site complications were not documented. ${ }^{8,10,19,20}$

\section{Evaluation of DSL}

As for the donor lower limb lymphedema, the overall incidence was 3 cases in a series of 189 patients (1.6\%). Two cases (aged 36 and 52 years) are reported by Vignes et al. ${ }^{16}$; the authors stated that lymphedema persisted for a median of 40 months post ALNT, but little information is provided about the clinical evaluation and documentation of these findings. Another case (age 52 years, BMI $24.0 \mathrm{~kg} / \mathrm{m}^{2}$ ) is reported by Pons et al. ${ }^{24}$; swelling of the thigh was observed 3 months postoperatively, and the symptoms were still present at the 24-month follow-up. Diagnosis of lymphedema was based on clinical evaluation, showing a 2-cm enlargement of the affected thigh, and lymphoscintigraphy that was performed before and after surgery; postoperatively, a delayed drainage of the affected lower limb was recorded.

\section{Statistical analysis}

According to our analysis, no significant correlations were found between the incidence of DSL and the recorded parameters (Table 2). More specifically, the patient's age, $\mathrm{BMI}$, or the follow-up period are not significantly related to the occurrence of DSL. The overall donor-site complications recorded postoperatively, the type of flap that was used, that is, combined reconstruction or LNT alone, as well as the main pedicle where the flap was based, did not affect the incidence of lower limb lymphedema $(p=0.312, p=0.552, p=1.000$, respectively). It is worth mentioning that all three patients who are reported to develop DSL, underwent a LNT with no simultaneous breast reconstruction. As for the number of harvested lymph nodes, there is no sufficient data correlating these variables with the incidence of DSL.

\section{Discussion}

During the last decades, significant progress concerning the surgical treatment of BCRL has been recorded and new techniques, namely microsurgical methods, have been 


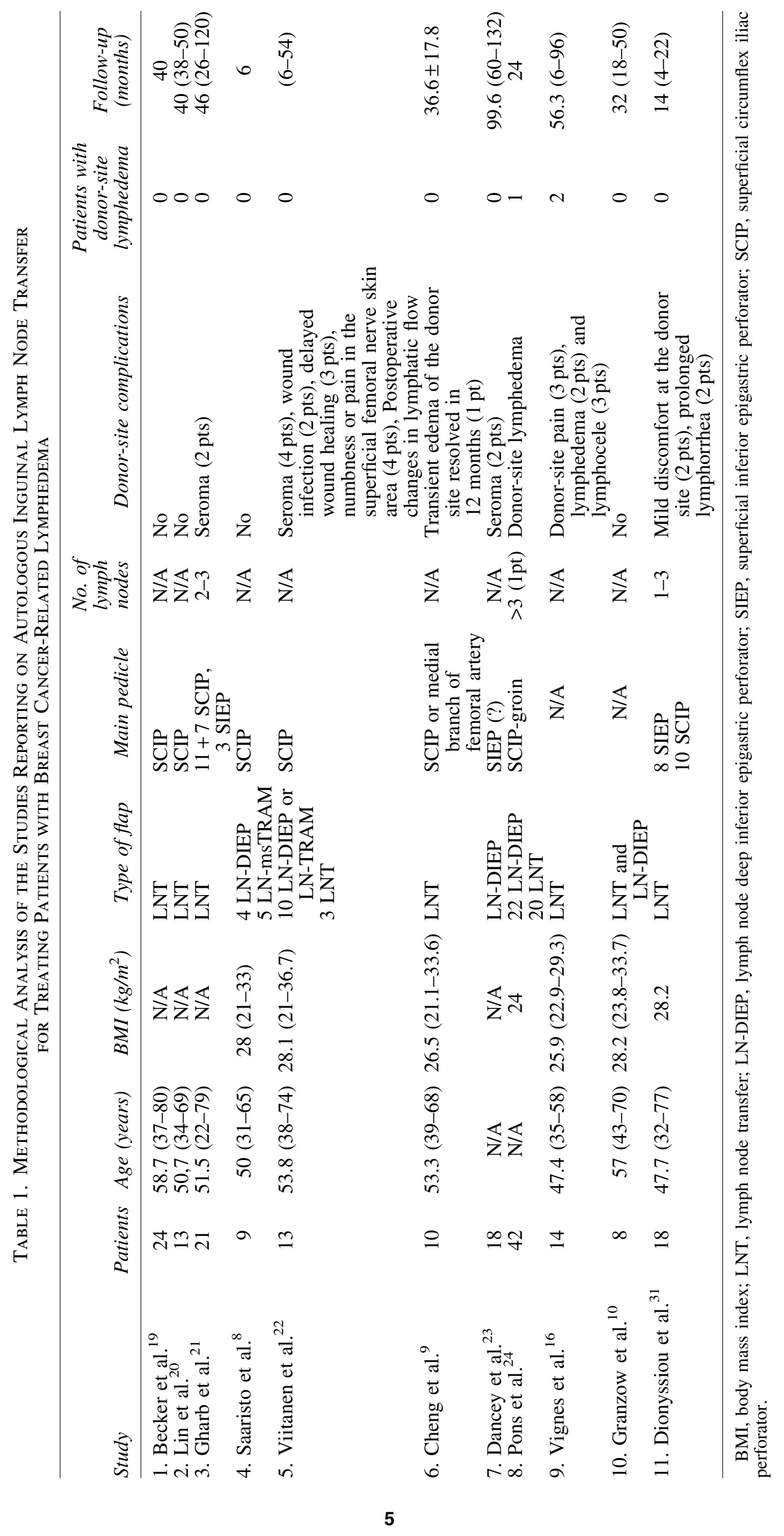


Table 2. Comparisons Between the Patients Who Presented Donor-Site Lymphedema Post Autologous Lymph Node Transfer and the Total Study Population

\begin{tabular}{lccc}
\hline & Patients with donor-site lymphedema & Total study population & $\mathrm{p}$ \\
\hline Age & 50.67 (SD: 2.31) & 54.14 (SD:11.77) & 0.088 \\
BMI & 27.67 (SD: 4.04) & 27.68 (SD: 1.75) & 0.644 \\
Main pedicle, $n(\%)$ & SCIP (100) & SCIP (75.2) & 1.000 \\
Flap type, $n(\%)$ & SIEP (24.8) & 0.552 \\
Donor-site complications, $n(\%)$ & LNT (100) & LNT-DIEP (34.3) & 0.312 \\
Follow-up & 33.3 & 11.7 & 0.839 \\
\hline
\end{tabular}

Precise data (SD or percentage) and $p$-values of both groups.

$\mathrm{BMI}$, body mass index; SD, standard deviation.

described. ${ }^{25,26}$ Among these procedures, ALNT provides a logical reconstructive option; a vascularized lymph node flap is harvested from the groin area and transferred to the affected axilla, after a mindful release of the recipient site's scar. This microsurgically revascularized functional lymph tissue flap not only works as a direct lymph pump that filters the excess fluid, but also releases growth factors (VEGF-C) in the nearby area promoting lymphangiogenesis and reconnection of the distal obstructed lymphatic system with the proximal one. ${ }^{8,27}$ Although a plethora of clinical studies have demonstrated promising results of ALNT, the risk of irreversible complications, mainly DSL related to the lymph flap harvesting, strongly attenuates the benefits of this technique.

In our systematic review on DSL, following ALNT in a homogeneous population of BCRL patients, we documented an incidence percentage of $1.6 \%$ ( 3 out of 189 cases). Trying to elucidate the impact of predisposing factors for DSL development, we recorded and analyzed several parameters that might have some etiological relation, that is, patients' BMI, type and main pedicle of the flap used, number of harvested lymph nodes, overall donor-site complications, as well as the follow-up period.

Although obesity is a risk factor for lymphedema development, no significant correlation was found in the three reported cases with DSL $(p=0.644)$. Similarly, the type of the lymph tissue flap (lymph node flap alone or combined abdominal flap), the occurrence of other donor-site morbidities, and the follow-up period were not shown to affect the induction of this dramatic complication.

Regarding the number of lymph nodes contained in the transferred flap, relevant data are not provided in the majority of studies analyzed in this review; however, it is worth pointing out that Vignes et al., who observed 2 cases of DSL in a series of 14 patients with BCRL, stated that they harvested the lymph node flap with an abundant amount of surrounding tissues. ${ }^{16}$ If we admit that this large amount of harvested soft tissue contains a large number of lymph nodes, we could assume that the high incidence of DSL reported in this series might be related to this parameter. Pons et al., in their reported cases with DSL, stated that at least three lymph nodes were embedded in the surrounding soft tissue flap. ${ }^{24}$ On the other hand, in the studies where a limited number of nodes (one to three) is reported to be transferred, postoperative DSL was not documented. ${ }^{18,21}$

It is well accepted that not only the number, but also the location of the harvested lymph nodes is significantly related to higher risks of inducing lower limb lymphedema, and several studies point out the importance of a careful lymph node selection to minimize the risk of disturbing the lymphatic circulation of the donor area. ${ }^{9,21,24,28}$ It is mandatory, therefore, to raise only lymphatic tissue that is located most laterally, and preserve not only the deep inguinal nodes but also the sentinel nodes that primarily drain the lower limb. ${ }^{22,29}$

The most laterally located lymph nodes included into the lymph flap are mainly nourished by the SCIA vascular axis, which constitutes the preferred pedicle in most studies; the SIEA vessels, although more medially located, have also been used to vascularize the lymph node flap without any DSL documentation. ${ }^{18,21}$ Regarding the reported DSL cases, in one patient, a superficial circumflex iliac perforator flap was transferred, ${ }^{24}$ whereas for the other two cases, no clear information is given by the authors.

For maximizing the safety of ALNT, new methods of mapping the lymph nodes preoperatively have been recently described, such as the use of the reverse lymphatic mapping and the magnetic resonance angiography. ${ }^{28,30}$ Intraoperative imaging of lymph nodes with Indocyanine Green dye has also been used to guide selective node harvesting and further improve the safety of the procedure. ${ }^{10}$ The "selected lymph node" technique using the SPECT-CT lymphoscintigraphy has been described aiming to identify preoperatively the most functional lymph nodes of the inguinal area and further reduce the donor-site morbidity. ${ }^{31}$ It is interesting to mention that none of the assessed studies reported to have used those specialized imaging techniques to evaluate and map pre- or postoperatively the donor-site lymphatics.

Regarding the postoperative documentation of DSL, only one of the reported DSL cases was evaluated with a postoperative lymphoscintigraphy, where a delayed lymphatic flow of the affected limb was recorded. ${ }^{24}$ In the other two cases, lower limb swelling was assessed only clinically, and no imaging tests were used to confirm the lymphedema diagnosis. Viitanen et al., in their published series of 12 BCRL patients, reported the use of postoperative lymphoscintigraphy of the donor-site limb after a lymphatic groin flap transfer during an 8- to 56-month follow-up. ${ }^{22}$ Interestingly, although the postoperative imaging tests revealed slower lymphatic flow of the donor-site lower limb in six patients, no one presented any clinical signs of swelling, or reported symptoms of heaviness, burden, or tension over the extremity; furthermore, no statistical difference between the circumference measurements of both lower limbs was recorded. 
It would be interesting to compare those findings with preoperative lymphoscintigraphy results if available, but unfortunately no data on lower limb lymphatic function before surgery was provided by the authors.

Scaglioni et al., recently published a comprehensive review on outcomes and complications of vascularized LNT from various donor sites; they reported that the inguinal area is the most frequently used donor site with a $10.9 \%$ overall complications rate, but similarly to our results, only $1.6 \%$ rate of DSL ${ }^{17}$; the second most commonly used donor site is the lateral thoracic area, which is related with significantly higher complication rates (15.8\% and $13.2 \%$, respectively). In this review, the authors present in detail the occurrence of complications following vascularized LNT from various donor sites, but they do not analyze the role of any causative factors, which is the aim of our study.

\section{Study Limitations}

Our systematic review has several limitations, mainly related to the lack of sufficient information regarding the patient's data, the surgical details of the technique used, and the pre- and postoperative evaluation of the lymphatic status of the donor area and ipsilateral lower limb; moreover, the number of articles that are assessed is not yet very large. Although significant correlations have not been extracted, it is obvious that both the preoperative planning of the procedure and the lymph node harvesting technique are of utmost importance, and, apparently, are implicated in the occurrence of DSL.

\section{Conclusion}

The results of this review clearly show that the percentage of DSL in BCRL patients following ALNT is low (1.6\%); nevertheless, when planning an ALNT, it is mandatory to avoid this most debilitating complication. The points that should be seriously considered to further maximize the safety of the procedure are (1) systematic preoperative evaluation of the lower limb lymphatic circulation with lymphoscintigraphy, (2) mindful selection of the inguinal nodes which may be harvested, (3) transfer of a small number of nodes $(<3)$ with limited surrounding lymph tissue, leaving intact the nodes that drain the lower limb, and (4) meticulous surgical technique avoiding extensive trauma of the inguinal area. A thorough preoperative clinical investigation of the patient is always essential ${ }^{18,32}$; a complete medical and family history is of great importance, while the examination of the patient's genetic profile may also be proposed in selected cases with a family history of lymphedema.

\section{Author Disclosure Statement}

No competing financial interests exist.

\section{References}

1. Togawa K, Ma H, Sullivan-Halley J, Neuhouser ML, Imayama I, Baumgartner KB, Smith AW, Alfano CM, McTiernan A, Ballard-Barbash R, Bernstein L. Risk factors for self-reported arm lymphedema among female breast cancer survivors: A prospective cohort study. Breast Cancer Res 2014; 16:414.
2. Golshan M, Smith B. Prevention and management of arm lymphedema in the patient with breast cancer. J Support Oncol 2006; 4:381-386.

3. McLaughlin SA, Wright MJ, Morris KT, Giron GL, Sampson MR, Brockway JP, Hurley KE, Riedel ER, Van Zee KJ. Prevalence of lymphedema in women with breast cancer 5 years after sentinel lymph node biopsy or axillary dissection: Objective measurements. J Clin Oncol 2008; 26:5213-5219.

4. Mortimer PS, Rockson SG. New developments in clinical aspects of lymphatic disease. J Clin Invest 2014; 124 : 915-921.

5. Cintolesi V, Stanton AW, Bains SK, Cousins E, Peters AM, Purushotham AD, Levick JR, Mortimer PS. Constitutively enhanced lymphatic pumping in the upper limbs of women who later develop breast cancer-related lymphedema. Lymphat Res Biol 2016; 14:50-61.

6. Rockson SG. The lymphatics and the inflammatory response: Lessons learned from human lymphedema. Lymphat Res Biol 2013; 11:117-120.

7. Rockson SG. Physiological mechanisms that predispose to the development of breast cancer-associated lymphedema full access. Lymphat Res Biol 2016; 14:49.

8. Saaristo AM, Niemi TS, Viitanen TP, Tervala TV, Hartiala $\mathrm{P}$, Suominen EA. Microvascular breast reconstruction and lymph node transfer for postmastectomy lymphedema patients. Ann Surg 2012; 255:468-473.

9. Cheng MH, Chen SC, Henry SL, Tan BK, Lin MC, Huang JJ. Vascularized groin lymph node flap transfer for postmastectomy upper limb lymphedema: Flap anatomy, recipient sites, and outcomes. Plast Reconstr Surg 2013; 131:1286-1298.

10. Granzow JW, Soderberg JM, Kaji AH, Dauphine C. An effective system of surgical treatment of lymphedema. Ann Surg Oncol 2014; 21:1189-1194.

11. Boccardo F, Fulcheri E, Villa G, Molinari L, Campisi C, Dessalvi S, Murdaca G, Campisi C, Santi PL, Parodi A, Puppo F, Campisi C. Lymphatic microsurgery to treat lymphedema: Techniques and indications for better results. Ann Plast Surg 2013; 71:191-195.

12. Becker C, Vasile JV, Levine JL, Batista BN, Studinger RM, Chen CM, Riquet M. Microlymphatic surgery for the treatment of iatrogenic lymphedema. Clin Plast Surg 2012; 39:385-398.

13. Becker C, Arrive L, Saaristo A, Germain M, Fanzio P, Batista BN, Piquilloud G. Surgical treatment of congenital lymphedema. Clin Plast Surg 2012; 39:377-384.

14. Chen HC, O’Brien BM, Rogers IW, Pribaz JJ, Eaton CJ. Lymph node transfer for the treatment of obstructive lymphoedema in the canine model. Br J Plast Surg 1990; 43:578-586.

15. Clodius L, Smith PJ, Bruna J, Serafin D. The lymphatics of the groin flap. Ann Plast Surg 1982; 9:447-458.

16. Vignes S, Blanchard M, Yannoutsos A, Arrault M. Complications of autologous lymph-node transplantation for limb lymphoedema. Eur J Vasc Endovasc Surg 2013; 45:516-520.

17. Scaglioni MF, Arvanitakis M, Chen YC, Giovanoli P, ChiaShen Yang J, Chang EI. Comprehensive review of vascularized lymph node transfers for lymphedema: Outcomes and complications. Microsurgery 2016; DOI:10.1002/ micr.30079.

18. Dionyssiou D, Demiri E, Tsimponis A, Sarafis A, Mpalaris V, Tatsidou G, Arsos G. A randomized control study of 
treating secondary stage II breast cancer-related lymphoedema with free lymph node transfer. Breast Cancer Res Treat 2016; 156:73-79.

19. Becker C, Assouad J, Riquet M, Hidden G. Postmastectomy lymphedema: Long-term results following microsurgical lymph node transplantation. Ann Surg 2006; 243:313-315.

20. Lin CH, Ali R, Chen SC, Wallace C, Chang YC, Chen HC, Cheng $\mathrm{MH}$. Vascularized groin lymph node transfer using the wrist as a recipient site for management of postmastectomy upper extremity lymphedema. Plast Reconstr Surg 2009; 123:1265-1275.

21. Gharb BB, Rampazzo A, Spanio di Spilimbergo S, Xu ES, Chung KP, Chen HC. Vascularized lymph node transfer based on the hilar perforators improves the outcome in upper limb lymphedema. Ann Plast Surg 2011; 67:589-593.

22. Viitanen TP, Mäki MT, Seppänen MP, Suominen EA, Saaristo AM. Donor-site lymphatic function after microvascular lymph node transfer. Plast Reconstr Surg 2012; 130:1246-1253.

23. Dancey A, Nassimizadeh A, Nassimizadeh M, Warner RM, Waters R. A chimeric vascularised groin lymph node flap and DIEP flap for the management of lymphoedema secondary to breast cancer. J Plast Reconstr Aesthet Surg 2013; 66:735-737.

24. Pons G, Masia J, Loschi P, Nardulli ML, Duch J. A case of donor-site lymphoedema after lymph node-superficial circumflex iliac artery perforator flap transfer. J Plast Reconstr Aesthet Surg 2014; 67:119-123.

25. Penha TR, Ijsbrandy C, Hendrix NA, Heuts EM, Voogd AC, von Meyenfeldt MF, van der Hulst RR. Microsurgical techniques for the treatment of breast cancer-related lymphedema: A systematic review. J Reconstr Microsurg 2013; 29:99-106.

26. Mehrara BJ, Zampell JC, Suami H, Chang DW. Surgical management of lymphedema: Past, present, and future. Lymphat Res Biol 2011; 9:159-167.
27. Tervala TV, Hartiala P, Tammela T, Visuri MT, YläHerttuala S, Alitalo K, Saarikko AM. Growth factor therapy and lymph node graft for lymphedema. J Surg Res 2015; 196:200-207.

28. Dayan JH, Dayan E, Smith ML. Reverse lymphatic mapping: A new technique for maximizing safety in vascularized lymph node transfer. Plast Reconstr Surg 2015; 135:277-285.

29. Granzow JW, Soderberg JM, Kaji AH, Dauphine C. Review of current surgical treatments for lymphedema. Ann Surg Oncol 2014; 21:1195-1201.

30. Dayan JH, Dayan E, Kagen A, Cheng MH, Sultan M, Samson W, Smith ML. The use of magnetic resonance angiography in vascularized groin lymph node transfer: An anatomic study. J Reconstr Microsurg 2014; 30:41-45.

31. Dionyssiou D, Demiri E, Arsos G, Tatsidou G, Pavlidis L. The "Selected Lymph Node" technique to facilitate lymph node flap harvest in the treatment of upper limb lymphoedema. Abstract Book of 26th EURAPS Annual Meeting, Edinburgh, May 2015, p. 147. Rome: EURAPS Central Office.

32. Sulo E, Hartiala $P$, Viitanen T, Mäki M, Seppänen M, Saarikko A. Risk of donor-site lymphatic vessel dysfunction after microvascular lymph node transfer. J Plast Reconstr Aesthet Surg 2015; 68:551-558.

Address correspondence to: Dimitrios Dionyssiou, MD, PhD Department of Plastic Surgery Faculty of Health Sciences School of Medicine Papageorgiou Hospital Aristotle University of Thessaloniki Periferiaki odos Thessalonikis Thessaloniki 56403 Greece

E-mail: ddionyssiou@gmail.com 